Journal of Organometallic Chemistry, 267 (1984) 229-236

Elsevier Sequoia S.A., Lausanne - Printed in The Netherlands

\title{
DARSTELLUNG UND EIGENSCHAFTEN VON DIPHENYLALKYLTHIO- UND DIPHENYLARYLTHIO-GALLANEN. KRISTALISTRUKTUR VON DIPHENYLETHYLTHIOGALLAN
}

GERHARD G. HOFFMANN * und CHRISTIAN BURSCHKA

Institut für Anorganische Chemie der Universität, Am Hubland D-8700 Würzburg (B.R.D.)

(Eingegangen den 27. Januar 1984)

\section{Summary}

Triphenylgallane reacts with alkyl- and aryl-thiols, respectively, with formation of the corresponding diphenylalkyl- and diphenylaryl-thiogallanes. Spectra and some physical and chemical properties of the new compounds are given. The results of the $\mathrm{X}$-ray structure determination of diphenylethylthiogallane are discussed.

\section{Zusammenfassung}

Triphenylgallan reagiert mit Alkyl- bzw. Arylthiolen unter Bildung der entsprechenden Diphenylalkyl- und Diphenylarylthiogallane. Spektren sowie einige physikalische und chemische Eigenschaften der neuen Verbindungen werden mitgeteilt. Die Ergebnisse der Röntgenstrukturuntersuchung von Diphenylethylthiogallan werden diskutiert.

\section{Einleitung}

In der Literatur sind nur wenige Reaktionen von Galliumorganylen mit schwefelhaltigen Substraten bekannt, bei denen eine Gallium-Schwefel-Bindung ausgebildet wird [1-6]. Die bisher einzigen Untersuchungen der Reaktivität von Triphenylgallan wurden in Dioxan als Lösungsmittel durchgeführt [3]; bei diesen Reaktionen liegt das Triphenylgallan naturgemäss als Etherat vor. In Anlehnung an diese Untersuchungen soll hier über einige Reaktionen von Triphenylgallan mit Alkyl- bzw. Arylthiolen in Benzol als Lösungsmittel berichtet werden, da in diesem Lösungsmittel Triphenylgallan monomer [7] und darüber hinaus nicht komplexiert vorliegt.

\section{Darstellung und Eigenschaften}

Triphenylgallan reagiert mit SH-aciden Alkyl- bzw. Arylthiolen bereitwillig und in guten Ausbeuten unter Abspaltung von Benzol nach

$$
\begin{aligned}
& \mathrm{Ph}_{3} \mathrm{Ga}+\mathrm{RSH} \stackrel{\text { Benzol }}{\longrightarrow} \mathrm{Ph}_{2} \mathrm{GaSR}+\mathrm{C}_{6} \mathrm{H}_{6} \\
& \left(\mathrm{R}=\mathrm{CH}_{3}, \mathrm{C}_{2} \mathrm{H}_{5}, \mathrm{n}-\mathrm{C}_{3} \mathrm{H}_{7}, \mathrm{i}-\mathrm{C}_{3} \mathrm{H}_{7}, \mathrm{t}-\mathrm{C}_{4} \mathrm{H}_{9}, \mathrm{Ph}, \mathrm{CH}_{2} \mathrm{Ph}\right)
\end{aligned}
$$


zu den gewünschten Diphenylalkylthio- bzw. Diphenylarylthiogallanen. Dabei kann merkliche Erwärmung der Reaktionsmischung beobachtet werden.

Den Reaktionsablauf kann man sich als Lewis-Säure-Base-Reaktion unter Bildung einer vierfach koordinierten Zwischenstufe vorstellen: diese Zwischenstufe reagiert allerdings sehr rasch unter Abspaltung von Benzol zum Endprodukt weiter (die Zwischenstufe konnte im Gegensatz zu den Reaktionen von Methylgalliumdiiodid mit Thiolen [6] weder 'H-NMR-spektroskopisch nachgewiesen, noch bei Raumtemperatur isoliert werden).

Alle auf diese Weise dargestellten Diphenylthiogallane sind relativ feuchtigkeitsund hydrolyseempfindliche, farblose Feststoffe, die, mit Ausnahme des t-Butylthioderivates, bei Temperaturen über $100^{\circ} \mathrm{C}$ schmelzen. Sie lösen sich mässig in chlorierten Kohlenwasserstoffen wie $\mathrm{CH}_{2} \mathrm{Cl}_{2}$ und $\mathrm{CHCl}_{3}$. Aus diesen Lösungen lassen sie sich leicht umkristallisieren (Diphenyl-t-butylthiogallan ist allerdings in diesen Lösungsmitteln nicht stabil!).

Osmometrische Molekulargewichtsbestimmungen der beschriebenen Verbindungen haben erstaunlicherweise gezeigt, dass die Alkylthioverbindungen zumindest in benzolischer Lösung trimere, die Phenylthio- bzw. Benzylthiogallane dagegen monomere Aggregate bilden (Tab. 1); dies steht im vollkommenen Gegensatz zu den Dimethylthiogallanen [2], die sowohl in Lösung als auch in der Gasphase dimere Strukturen haben.

Einige physikalische Eigenschaften sowie die Ausbeuten sind in Tab. 1 zusammengestellt.

Die Thiogallane reagieren alle begierig mit Alkoholen, zunächst unter Austausch der Thiogruppierung, gegen die Alkoholatreste. Dies lässt sich mit Hilfe des Pearson'schen Prinzips [8] über harte und weiche Säuren und Basen zufriedenstellend erklären, nach dem $\mathrm{Ga}^{3+}$-Ionen als harte Lewis-Säure zu betrachten sind. Mit einem Überschuss Alkohol werden erwartungsgemäss auch die Phenylgruppen am Gallium substituiert.

TABELLE 1

DATEN ZU DEN THIOGALLANEN

\begin{tabular}{|c|c|c|c|c|c|c|c|c|}
\hline & \multirow{2}{*}{$\begin{array}{l}\text { Summenformel } \\
\text { (Molmasse) }\end{array}$} & \multirow{2}{*}{$\begin{array}{l}\text { Ausb. } \\
\text { (\%) }\end{array}$} & \multirow{2}{*}{$\begin{array}{l}\text { MP } \\
\left({ }^{\circ} \mathrm{C}\right)\end{array}$} & \multirow{2}{*}{$\begin{array}{l}\text { Molmasse }{ }^{a} \\
\text { (AG) }^{b}\end{array}$} & \multicolumn{4}{|c|}{ Analyse (Gef. (ber.)(\%)) } \\
\hline & & & & & $\mathrm{C}$ & $\mathbf{H}$ & $\mathrm{Ga}$ & $\mathbf{S}$ \\
\hline II & $\begin{array}{l}\mathrm{C}_{13} \mathrm{H}_{13} \mathrm{GaS} \\
(271.0)\end{array}$ & 76 & 223 & $\begin{array}{l}796 \\
(3)\end{array}$ & $\begin{array}{l}57.7 \\
(57.61)\end{array}$ & $\begin{array}{l}4.60 \\
(4.86)\end{array}$ & $\begin{array}{l}25.1 \\
(25.73)\end{array}$ & $\begin{array}{l}11.5 \\
(11.83)\end{array}$ \\
\hline III & $\begin{array}{l}\mathrm{C}_{14} \mathrm{H}_{15} \mathrm{GaS} \\
(285.0)\end{array}$ & 92 & 186 & $\begin{array}{l}848 \\
(3)\end{array}$ & $\begin{array}{l}58.9 \\
(58.99)\end{array}$ & $\begin{array}{c}5.17 \\
(5.30)\end{array}$ & $\begin{array}{l}24.7 \\
(24.46)\end{array}$ & $\begin{array}{l}11.4 \\
(11.25)\end{array}$ \\
\hline IV & $\begin{array}{l}\mathrm{C}_{15} \mathrm{H}_{17} \mathrm{GaS} \\
(299.1)\end{array}$ & 87 & 110 & $\begin{array}{l}912 \\
\text { (3) }\end{array}$ & $\begin{array}{l}60.6 \\
(60.24)\end{array}$ & $\begin{array}{c}5.63 \\
(5.73)\end{array}$ & $\begin{array}{l}23.2 \\
(23.31)\end{array}$ & $\begin{array}{l}10.5 \\
(10.72)\end{array}$ \\
\hline $\mathbf{v}$ & $\begin{array}{l}\mathrm{C}_{15} \mathrm{H}_{17} \mathrm{GaS} \\
(299.1)\end{array}$ & 94 & 146 & $\begin{array}{l}954 \\
(3)\end{array}$ & $\begin{array}{l}59.9 \\
(60.24)\end{array}$ & $\begin{array}{c}5.55 \\
(5.73)\end{array}$ & $\begin{array}{l}22.9 \\
(23.31)\end{array}$ & $\begin{array}{c}10.3 \\
(10.72)\end{array}$ \\
\hline VI & $\begin{array}{l}\mathrm{C}_{16} \mathrm{H}_{19} \mathrm{GaS} \\
(313.1)\end{array}$ & 91 & 92 & - & $\begin{array}{l}61.4 \\
(61.37)\end{array}$ & $\begin{array}{c}5.93 \\
(6.12)\end{array}$ & $\begin{array}{l}22.8 \\
(22.27)\end{array}$ & $\begin{array}{l}10.0 \\
(10.24)\end{array}$ \\
\hline VII & $\begin{array}{l}\mathrm{C}_{18} \mathrm{H}_{15} \mathrm{GaS} \\
(333.1)\end{array}$ & 93 & 174 & $\begin{array}{l}340 \\
(1)\end{array}$ & $\begin{array}{c}64.9 \\
(64.90)\end{array}$ & $\begin{array}{c}4.56 \\
(4.54)\end{array}$ & $\begin{array}{c}21.4 \\
(20.93)\end{array}$ & $\begin{array}{c}9.7 \\
(9.63)\end{array}$ \\
\hline VIII & $\begin{array}{l}\mathrm{C}_{19} \mathrm{H}_{17} \mathrm{GaS} \\
(347.1)\end{array}$ & 99 & 137 & $\begin{array}{l}331 \\
\text { (1) }\end{array}$ & $\begin{array}{c}65.2 \\
(65.74)\end{array}$ & $\begin{array}{c}4.89 \\
(4.94)\end{array}$ & $\begin{array}{l}18.9 \\
(20.09)\end{array}$ & $\begin{array}{c}9.1 \\
(9.24)\end{array}$ \\
\hline
\end{tabular}

"Dampfdruckosmometrisch in Benzol. ${ }^{b}$ Assoziationsgrad. 
Kristallstruktur von Diphenylethylthiogallan

Sind Angaben zur Synthese eines Verbindungstyps bisher so spärlich dokumentiert wie im Fall der Organothiogallane, so kann es nicht überraschen, wenn Details ihrer Stereochemie unbekannt sind. Hinweise auf ihren molekularen Aufbau erhielt man allerdings schon 1953 [2] bei ebullioskopischen Molgewichtsbestimmungen und solchen in der Gasphase an Produkten der Zusammensetzung $\mathrm{Me}_{2} \mathrm{GaXR}$ $\left(\mathrm{X}=\mathrm{O}, \mathrm{S}, \mathrm{Se} ; \mathrm{R}=\mathrm{CH}_{3}, \mathrm{C}_{6} \mathrm{H}_{5}\right.$ ), die als Dimere erkannt wurden und für die eine 4-Ring-Struktur formuliert werden kann, in der $\mathrm{Ga}$ und $\mathrm{X}$ alternieren. Derartige Vierer-Zyklen, in denen das Illb-Metall seine Koordinationszahl auf 4 oder sogar auf 5 [9] erhöht, sind mit Stickstoff oder Sauerstoff als Ligand X inzwischen röntgenographisch belegt, so dass auch für entsprechende Thiogallane im Kristall ein solcher Aufbau erwartet werden kann. Allerdings ist auch die Bildung höherer Molekülaggregate möglich, wie die tetramere Struktur von Dimethylgalliumhydroxid [10] beweist. Da die in Benzol ermittelten Molmassen in letzterem Fall jedoch das Trimere hatten erwarten lassen, schien uns eine Prognose aufgrund der in Tab. 1 aufgeführten Molmassen bei den hier vorgestellten Verbindungen unsicher.

Für Diffraktometermessungen geeignete Einkristalle wurden aus Methylenchlorid erhalten. Die Untersuchung eines ca. $0.2 \times 0.3 \times 0.3 \mathrm{~mm}$ grossen Einkristallbruchstücks (Syntex-P2 ${ }_{1}$-Diffraktometer, Mo- $K_{\alpha}$-Strahlung, Graphit-Monochromator, $\lambda$ $0.71069 \AA$ ) führten zu einer monoklinen Elementarzelle, die Raum für 4 Formeleinheiten $\mathrm{C}_{2} \mathrm{H}_{5} \mathrm{SGa}\left(\mathrm{C}_{6} \mathrm{H}_{5}\right)_{2}$ bietet $\left(\rho_{\mathrm{ro}} 1.46 ; \rho_{\exp } 1.44 \mathrm{mg} \mathrm{mm}^{-3}\right)$. Aufgrund von Zersetzungserscheinungen bei Bestrahlung wurden die Kristalle in einer Glaskapillare unter Schutzgas montiert und für die Datensammlung auf ca. $173 \mathrm{~K}$ gekühlt. Bei dieser Temperatur lieferte eine least-squares-Rechnung, basierend auf 13 zentrierten Reflexen im Bereich von $2 \theta=13$ bis $17^{\circ}$, die Gitterkonstanten $a$ 1087.3(4), $b$ $1409.4(4), c 855.6(4) \mathrm{pm}, \beta$ 97.39(2) ${ }^{\circ}, V 1300.3 \times 10^{6} \mathrm{pm}^{2}$. Die Vermessung von 1896 Beugungsintensitäten im Bereich $2 \theta=5-45^{\circ}$ ( $\omega$-scan, $\Delta \omega=0.8^{\circ}$ ) ergab 1701 symmetrieunabhängige Reflexe, von denen 1593 das Kriterium $I_{0} \geqslant 3 \sigma\left(I_{0}\right)$ erfüllten. Sie wurden nach der $\psi$-scan Methode bezüglich Absorption korrigiert $\left(\mu_{\mathrm{Mo}} 2.35 \mathrm{~mm}^{-1}\right)$.

Als Raumgruppe konnte $P 2_{1} / c$ aufgrund der systematischen Auslöschungen erkannt werden. Nachdem in einer Patterson-Synthese das Ga-Atom gefunden war, liessen sich Schwefel, Kohlenstoff und ein Teil der H-Atome mittels Fourier- und Differenzfourier-Synthesen lokalisieren. Die restlichen H-Atome wurden auf idealisierten Positionen in das Modell eingefügt, jedoch nicht verfeinert. Nach Optimierung von 145 Variablen über mehrere least-squares-Zyklen ergaben sich für 1593 (1701) Reflexe $R$-Werte von $R_{1}=0.031(0.033)$ und $R_{2}=0.039(0.039)$. Eine Differenz-Fourier-Synthese enthielt nur Maxima $=0.5 \mathrm{e} / \mathrm{pm} \times 10^{6}$. Die bei diesem Stand erhaltenen Atomkoordinaten sind zusammen mit $B_{\text {eq }}$-Werten in Tab. 2 angegeben, Tab. 3 enthält eine Auswahl der resultierenden Atomabstände und Winkel. Alle Rechnungen erfolgten mit Programmen des XTL-Systems [11] unter Verwendung von Strukturfaktoren für ungeladene Atome [12].

Erstaunlicherweise zeigt das Ergebnis der Analyse (vgl. Fig. 1) ein zentrosymmetrisches zyklisches Dimeres mit exakt planarem Ga-S-Ga-S-Vierring. Ungewöhnliche intermolekulare Abstände sind nicht festzustellen. Die Koordinationsgeometrie des Ga-Atoms ist gegenüber einem idealen Tetraeder merklich verzerrt, und zwar in gleichem Sinne wie im Vierring mit $X=N$ [13] (C-Ga-C 124.0, N-Ga-N 82.1 ${ }^{\circ}$ ) oder im tetrameren Hydroxid [10] (C-Ga-C 129.2, O-Ga-O 98.8 ${ }^{\circ}$. Die Ebene $\mathrm{C}(1) \mathrm{GaC}(7)$ steht erwartungsgemäss annähernd rechtwinklig 
TABELLE 2

ATOMKOORDINATEN UND ISOTROPE TEMPERATURFAKTOREN $\left(\mathrm{pm} \times 10^{6}\right)$ IN DER KRISTALLSTRUKTUR VON DIPHENYLETHYLTHIOGALLAN

\begin{tabular}{|c|c|c|c|c|}
\hline Atom & $x$ & $y$ & $z$ & $B_{\text {ey }}$ \\
\hline $\mathbf{G a}$ & $0.35335(4)$ & $0.48502(3)$ & $0.43769(5)$ & $1.99(2)$ \\
\hline $\mathbf{S}$ & $0.53420(9)$ & $0.39784(7)$ & $0.4013(1)$ & $2.10(4)$ \\
\hline$C(1)$ & $0.2729(3)$ & $0.5477(3)$ & $0.2464(4)$ & $2.2(2)$ \\
\hline$C(2)$ & $0.2126(4)$ & $0.4958(3)$ & $0.1218(5)$ & $2.6(2)$ \\
\hline$C(3)$ & $0.1502(4)$ & $0.5407(3)$ & $-0.0104(5)$ & $3.0(2)$ \\
\hline$C(4)$ & $0.1464(4)$ & $0.6371(3)$ & $-0.0190(5)$ & $3.1(2)$ \\
\hline$C(5)$ & $0.2054(4)$ & $0.6914(3)$ & $0.1033(5)$ & $3.1(2)$ \\
\hline$C(6)$ & $0.2680(4)$ & $0.6466(3)$ & $0.2345(5)$ & $2.6(2)$ \\
\hline$C(7)$ & $0.2576(3)$ & $0.4073(3)$ & $0.5695(4)$ & $2.1(2)$ \\
\hline$C(8)$ & $0.1290(4)$ & $0.4127(3)$ & $0.5495(5)$ & $2.7(2)$ \\
\hline$C(9)$ & $0.0596(4)$ & $0.3596(3)$ & $0.6429(5)$ & $3.4(2)$ \\
\hline$C(10)$ & $0.1169(4)$ & $0.3003(3)$ & $0.7566(5)$ & $3.4(2)$ \\
\hline$C(11)$ & $0.2425(4)$ & $0.2921(3)$ & $0.7781(5)$ & $2.9(2)$ \\
\hline$C(12)$ & $0.3132(4)$ & $0.3458(3)$ & $0.6843(5)$ & $2.5(2)$ \\
\hline$C(13)$ & $0.5702(4)$ & $0.4285(3)$ & $0.2033(5)$ & $3.0(2)$ \\
\hline$C(14)$ & $0.5102(4)$ & $0.3628(3)$ & $0.0809(5)$ & $2.9(2)$ \\
\hline$H(2)$ & $0.2119(0)$ & $0.4256(0)$ & $0.1267(0)$ & $3.0(0)$ \\
\hline $\mathbf{H}(3)$ & $0.1075(0)$ & $0.5036(0)$ & $-0.0965(0)$ & $3.0(0)$ \\
\hline$H(4)$ & $0.1042(0)$ & $0.6700(0)$ & $-0.1118(0)$ & $3.0(0)$ \\
\hline$H(5)$ & $0.2024(0)$ & $0.7599(0)$ & $0.0982(0)$ & $3.0(0)$ \\
\hline$H(6)$ & $0.3083(0)$ & $0.6862(0)$ & $0.3219(0)$ & $3.0(0)$ \\
\hline$H(8)$ & $0.0865(0)$ & $0.4547(0)$ & $0.4674(0)$ & $3.0(0)$ \\
\hline$H(9)$ & $-0.0321(0)$ & $0.3637(0)$ & $0.6273(0)$ & $3.0(0)$ \\
\hline$H(10)$ & $0.0666(0)$ & $0.2631(0)$ & $0.8223(0)$ & $3.0(0)$ \\
\hline$H(11)$ & $0.2825(0)$ & $0.2499(0)$ & $0.8569(0)$ & $3.0(0)$ \\
\hline $\mathrm{H}(12)$ & $0.4050(0)$ & $0.3392(0)$ & $0.7027(0)$ & $3.0(0)$ \\
\hline$H(131)$ & $0.5532(0)$ & $0.4954(0)$ & $0.1899(0)$ & $3.0(0)$ \\
\hline $\mathbf{H}(132)$ & $0.6678(0)$ & $0.4174(0)$ & $0.2102(0)$ & $3.0(0)$ \\
\hline$H(141)$ & $0.5417(0)$ & $0.3724(0)$ & $-0.0235(0)$ & $3.0(0)$ \\
\hline$H(142)$ & $0.4257(0)$ & $0.3712(0)$ & $0.0592(0)$ & $3.0(0)$ \\
\hline$H(143)$ & $0.5266(0)$ & $0.3015(0)$ & $0.1041(0)$ & $3.0(0)$ \\
\hline
\end{tabular}

$\left(87.2^{\circ}\right)$ zur Ebene des Vierrings, wobei die $\mathrm{Ga}-\mathrm{C}$-Bindungslängen gut mit den bei [10] und [13] gefundenen Werten übereinstimmen. Für den $\mathrm{Ga}-\mathrm{S}$-Bindungsabstand von im Mittel 238 pm existieren bisher nur wenige Vergleichswerte. In der Kristallstruktur von $\mathrm{I}_{2} \mathrm{GaSMe}$ [14], die ebenfalls einen $\mathrm{Ga}-\mathrm{S}-\mathrm{Ga}-\mathrm{S}-$ Vierring enthält, haben

TABELLE 3

AUSWAHL DER INTERATOMAREN ABSTÄNDE (pm) UND WINKEL $\left({ }^{\circ}\right)$ IN DER KRISTALL. STRUKTUR VON DIPHENYLETHYLTHIOGALLAN

\begin{tabular}{llll}
\hline$G a-S$ & $237.3(1)$ & $S-G a-S^{\prime}$ & $93.58(4)$ \\
$G a-S^{\prime}$ & $238.4(1)$ & $G a-S-G a^{\prime}$ & $86.42(3)$ \\
$G a-C(1)$ & $196.4(4)$ & $\mathrm{Cl}-\mathrm{Ga}-\mathrm{C}(7)$ & $121.2(2)$ \\
$\mathrm{Ga}-\mathrm{C}(7)$ & $196.4(4)$ & $\mathrm{S}-\mathrm{Ga}-\mathrm{C}(1)$ & $108.1(1)$ \\
$\mathrm{S}-\mathrm{C}(13)$ & $183.8(4)$ & $\mathrm{S}-\mathrm{Ga}-\mathrm{C}(7)$ & $109.0(1)$ \\
$\mathrm{C}(13)-\mathrm{C}(14)$ & $148.5(6)$ & $\mathrm{Ga}-\mathrm{S}-\mathrm{C}(13)$ & $106.1(1)$ \\
$\mathrm{C}-\mathrm{C}_{\text {phenyl }}$ & $135.9(6)-139.8(6)$ & $\mathrm{Ga}-\mathrm{S}-\mathrm{C}(13)$ & $102.4(1)$ \\
\hline
\end{tabular}




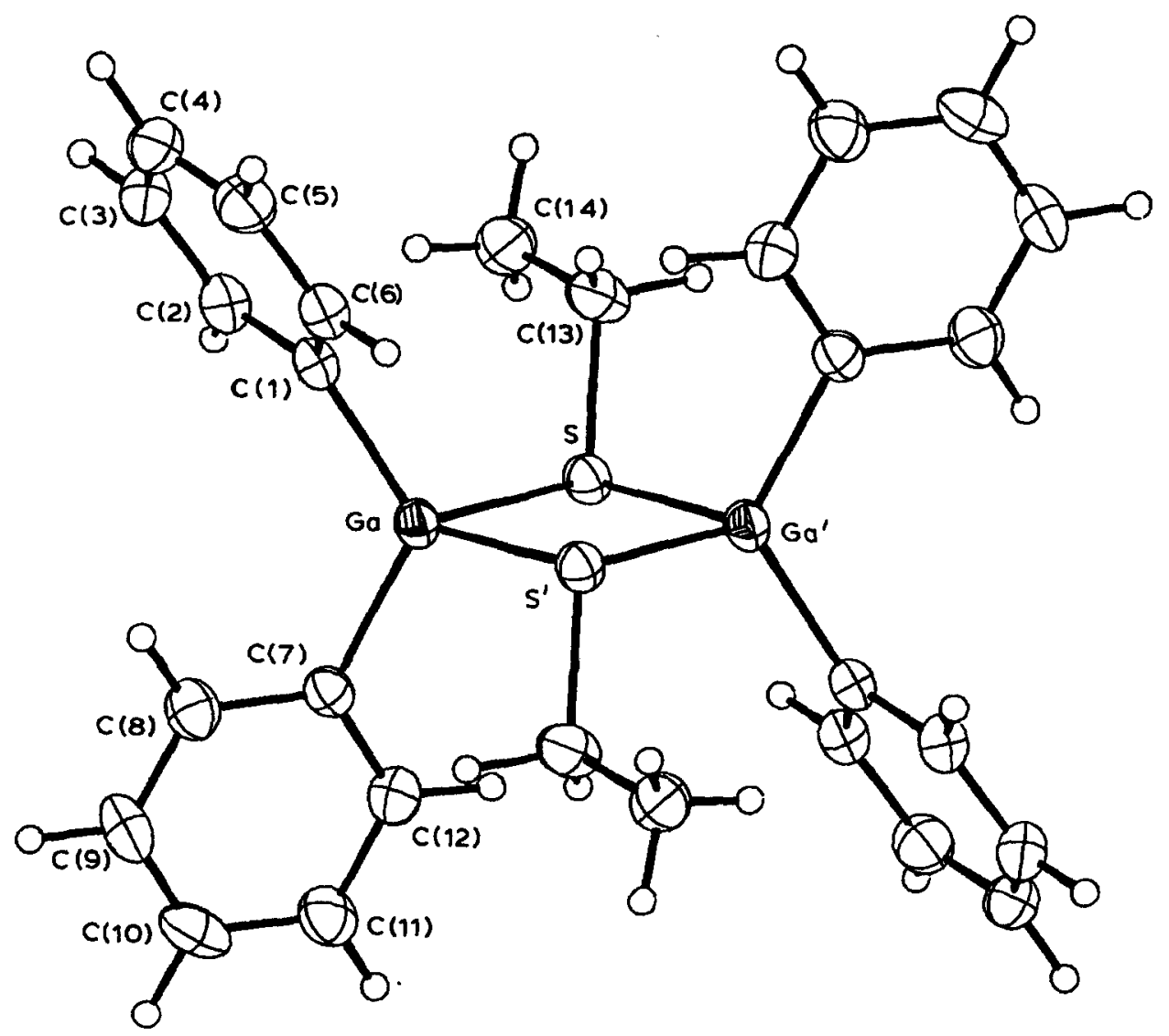

Fig. 1. ORTEP-Plot der Molekülstruktur von Diphenylethylthiogallan im Kristall.

wir geringfügig kleinere Abstände von 234-235 pm festgestellt. Dagegen sind auch schon Werte von $233 \mathrm{pm}$ bei Bis(dimethylgallium)- $N, N^{\prime}$-dimethyldithiooxamid [15] und 241-247 pm bei $\mathrm{Ga}\left(\mathrm{S}_{2} \mathrm{CNEt}_{2}\right)_{3}$ [16] ermittelt worden.

Bemerkenswert erscheint der relativ kleine Winkel Ga-S-Ga von $86.42(3)^{\circ}$.

\section{Spektroskopische Betrachtungen}

Die ${ }^{1}$ H-NMR-Spektren der Verbindungen sind in Tab. 4 zusammengefasst. Allgemein lässt sich feststellen, dass die Signale der Alkylgruppen der Thiogallane im Vergleich zu den Signalen der Alkylgruppen der freien Thiole nach tieferem Feld verschoben sind, was auf eine stärkere Entschirmung hindeutet. Auch eine Verbreiterung der Signale lässt sich erkennen.

In den Infrarotspektren (Tab. 5) liegen die antisymmetrischen KohlenstoffSchwefel-Schwingungen bei $730 \mathrm{~cm}^{-1}$; die Gallium-Schwefel-Streckschwingungen lassen sich bei $325-315$ und $255-245 \mathrm{~cm}^{-1}$ zuordnen. 
TABELLE 4

${ }^{1} \mathrm{H}$-NMR-DATEN DER THIOGALLANE ( $J$ $8 \mathrm{~Hz}$ )

\begin{tabular}{llll}
\hline Ver. & $\delta(\mathrm{ppm})$ & Zuordnung & Lösungsmittel/Standard \\
\hline II & $7.73-7.03 \mathrm{~m}$ & $\mathrm{Ph}$ & $\mathrm{CH}_{2} \mathrm{Cl}_{2} / \mathrm{CH}_{2} \mathrm{Cl}_{2}$ \\
& $1.77 \mathrm{~s}$ & $\mathrm{SCH}$ & \\
III & $7.77-7.17 \mathrm{~m}$ & $\mathrm{Ph}$ & $\mathrm{CH}_{2} \mathrm{Cl}_{2} / \mathrm{CH}_{2} \mathrm{Cl}_{2}$ \\
& $2.67 \mathrm{q}$ & $\mathrm{SCH}_{2}$ & \\
& $0.88 \mathrm{t}$ & $\mathrm{CH}_{3}$ & \\
IV & $7.77-7.17 \mathrm{~m}$ & $\mathrm{Ph}$ & $\mathrm{CH}_{2} \mathrm{Cl}_{2} / \mathrm{CH}_{2} \mathrm{Cl}_{2}$ \\
& 2.31 & $\mathrm{SCH}_{2}$ & \\
& $1.23 \mathrm{q}$ & $\mathrm{CH}_{2}$ & \\
& $0.53 \mathrm{t}$ & $\mathrm{CH}_{3}$ & \\
V & $7.83-7.20 \mathrm{~m}$ & $\mathrm{Ph}$ & $\mathrm{CH}_{2} \mathrm{Cl}_{2} / \mathrm{CH}_{2} \mathrm{Cl}_{2}$ \\
& $3.13 \mathrm{sep}$ & $\mathrm{SCH}$ & \\
& $0.97 \mathrm{~d}$ & $\mathrm{CH}_{3}$ & \\
VI & $7.83-7.20 \mathrm{~m}$ & $\mathrm{Ph}$ & $\mathrm{CH}_{2} \mathrm{Cl}_{2} / \mathrm{CH}_{2} \mathrm{Cl}_{2}$ \\
& $1.20 \mathrm{~s}$ & $\mathrm{CH}_{3}$ & \\
VII & $7.63-6.93 \mathrm{~m}$ & $\mathrm{Ph}$ & $\mathrm{CH}_{2} \mathrm{Cl}_{2} / \mathrm{CH}_{2} \mathrm{Cl}_{2}$ \\
VII & $7.63-6.37 \mathrm{~m}$ & $\mathrm{Ph}$ & $\mathrm{CS}_{2} / \mathrm{TMS}$ \\
& $3.57 \mathrm{~s}$ & $\mathrm{SCH}$ & \\
\hline
\end{tabular}

TABELLE 5

IR-DATEN DER THIOGALLANE $\left(\mathrm{cm}^{-1}\right)$

\begin{tabular}{|c|c|c|c|c|c|c|c|}
\hline II & III & IV & $\mathbf{V}$ & VI & VII & VIII & $\nu$ \\
\hline $996 \mathrm{~m}$ & $995 \mathrm{~m}$ & $996 \mathrm{~m}$ & $996 \mathrm{~m}$ & $997 \mathrm{~m}$ & $996 \mathrm{~m}$ & $996 \mathrm{~m}$ & \\
\hline $966 \mathrm{~m}$ & $968 \mathrm{~m}$ & & $972 w$ & $972 w$ & $911 w$ & $977 w$ & \\
\hline \multirow[t]{3}{*}{$856 w$} & $854 w$ & $891 w$ & $879 w$ & $858 w$ & & $858 w$ & \\
\hline & & $786 w$ & & $807 w$ & & $799 w$ & \\
\hline & & $761 w$ & & & $745 \mathrm{~m}$ & $765 \mathrm{~m}$ & \\
\hline $731 \mathrm{~s}$ & $737 \mathrm{~s}$ & & $732 s$ & $735 \mathrm{~s}$ & $733 \mathrm{~s}$ & & \\
\hline $724 s$ & $728 \mathrm{~s}$ & $728 \mathrm{~s}$ & $728 s$ & $730 \mathrm{~s}$ & $721 \mathrm{~s}$ & $727 \mathrm{~s}$ & $\nu(\mathrm{CS})$ \\
\hline $697 \mathrm{~s}$ & $703 \mathrm{~s}$ & $705 \mathrm{sh}$ & $703 \mathrm{~s}$ & $698 \mathrm{~s}$ & $698 \mathrm{~s}$ & $694 s$ & \\
\hline $687 \mathrm{~m}$ & $700 \mathrm{~s}$ & $700 s$ & $700 \mathrm{~s}$ & & $685 \mathrm{~s}$ & & \\
\hline $667 \mathrm{~m}$ & $666 \mathrm{~m}$ & $668 \mathrm{~m}$ & $668 \mathrm{~m}$ & $672 \mathrm{~m}$ & $672 \mathrm{~m}$ & $667 \mathrm{~m}$ & \\
\hline \multirow[t]{4}{*}{$658 w$} & $658 w$ & $658 w$ & $657 w$ & $664 w$ & & & \\
\hline & $647 \mathrm{~m}$ & $633 w$ & $609 \mathrm{~m}$ & & $615 \mathrm{~m}$ & $615 \mathrm{~m}$ & \\
\hline & & & & $572 \mathrm{~m}$ & & $569 w$ & \\
\hline & & & & & $481 w$ & $564 \mathrm{~m}$ & \\
\hline \multirow[t]{5}{*}{$452 \mathrm{~s}$} & $452 \mathrm{~s}$ & $455 \mathrm{~s}$ & $453 \mathrm{~s}$ & $452 \mathrm{~s}$ & $457 \mathrm{~s}$ & $454 \mathrm{~s}$ & \\
\hline & & $449 s$ & 449sh & & $449 s$ & $448 \mathrm{~s}$ & \\
\hline & & & $430 w$ & $420 \mathrm{~m}$ & $429 w$ & & \\
\hline & $370 \mathrm{~m}$ & $383 \mathrm{~m}$ & $364 s$ & $391 \mathrm{~m}$ & $392 w$ & $390 w$ & \\
\hline & & & & $349 \mathrm{~m}$ & 345sh & & \\
\hline $323 \mathrm{~s}$ & $319 \mathrm{~s}$ & $322 \mathrm{~s}$ & $320 \mathrm{~s}$ & $323 s$ & $325 \mathrm{~m}$ & $319 \mathrm{~m}$ & $\nu(\mathrm{GaS})$ \\
\hline $290 \mathrm{~s}$ & $288 \mathrm{~m}$ & $295 \mathrm{sh}$ & & $304 s$ & $318 \mathrm{~m}$ & 280 s & \\
\hline \multirow[t]{2}{*}{$281 \mathrm{~s}$} & & $279 \mathrm{~m}$ & & & $277 \mathrm{~m}$ & & \\
\hline & & & & & $263 \mathrm{~m}$ & & \\
\hline $247 s$ & $253 \mathrm{~m}$ & $245 w$ & $256 s$ & $251 \mathrm{~s}$ & $248 w$ & $246 \mathrm{~m}$ & $\nu(\mathrm{GaS})$ \\
\hline $235 w$ & & & $245 s h$ & $245 s$ & & & \\
\hline $209 w$ & & & & $228 w$ & 220sh & $210 \mathrm{~m}$ & \\
\hline
\end{tabular}




\section{Experimentelles}

IR-Spektren wurden als Nujolverreibung auf CsI-Platten am Gerät Perkin-Elmer 283 und 'H-NMR-Spektren am Gerăt Varian T 60 aufgenommen. Die Schmelzpunkte wurden am Gerät Du Pont 990 Thermal Analyser, die C,H-Analysen am Gerät Carlo Erba 1106, Schwefel nach Schöniger [17] und Gallium komplexometrisch [18] bestimmt. Molmassen: Gerät Knauer Dampfdruckosmometer. Alle Arbeiten wurden unter $\mathrm{N}_{2}$-Atmosphäre in getrockneten Lösungsmitteln ausgeführt. Triphenylgallan [19] wurde nach dem in der Literatur bekannten Verfahren synthetisiert.

\section{Diphenylmethylthiogallan (II)}

In eine Lösung von $1.47 \mathrm{~g}(4.88 \mathrm{mmol})$ Triphenylgallan (I) in $20 \mathrm{ml}$ Benzol werden $109 \mathrm{~cm}^{3}(4.88 \mathrm{mmol})$ Methanthiol-Gas eingeleitet. Nach ca. $10 \mathrm{~min}$ fällt ein weisser Feststoff aus. Es wird noch 30 min gerührt, das Lösungsmittel abgezogen, in Methylenchlorid aufgenommen und kristallisieren lassen. Ausbeute: $1.00 \mathrm{~g}$ (75.8\%) farblose Kristalle.

\section{Diphenylethylthiogallan (III)}

Zu einer Lösung von $1.50 \mathrm{~g}(4.98 \mathrm{mmol}) \mathrm{I}$ in $10 \mathrm{ml}$ Benzol wird eine Lösung von $0.310 \mathrm{~g}(5.00 \mathrm{mmol})$ Ethanthiol in ca. $10 \mathrm{ml}$ Benzol getropft, dabei ist eine leicht exotherme Reaktion zu beobachten. Es wird noch $30 \mathrm{~min}$ gerührt, das Lösungsmittel abgezogen, in Methylenchlorid aufgenommen und kristallisieren lassen. Ausbeute: $1.30 \mathrm{~g}(91.5 \%)$ farblose Nadeln.

\section{Diphenyl-n-propylthiogallan (IV)}

Durchführung und Aufarbeitung wie III. Ansatz: $1.50 \mathrm{~g}(4.98 \mathrm{mmol})$ I und 0.379 $\mathrm{g}(4.98 \mathrm{mmol}) \mathrm{n}$-Propanthiol. Ausbeute: $1.30 \mathrm{~g}(87.2 \%)$ farblose Kristalle.

\section{Diphenyl-i-propylthiogallan (V)}

Durchführung und Aufarbeitung wie III. Ansatz: $1.50 \mathrm{~g}$ (4.98 mmol) I und 0.380 $\mathrm{g}$ (5.00 mmol) i-Propanthiol. Ausbeute: $1.40 \mathrm{~g}$ (94.0\%) farblose Kristalle.

Diphenyl-t-butylthiogallan (VI)

Durchführung wie III. Das Lösungsmittel wird abgezogen, der Rückstand mit $\mathrm{n}$-Hexan gewaschen und getrocknet. Ansatz: $1.90 \mathrm{~g}(6.31 \mathrm{mmol}) \mathrm{I}$ und $0.560 \mathrm{~g}(6.31$ mmol) t-Butanthiol. Ausbeute: $1.80 \mathrm{~g}$ (90.9\%) weisses, mikrokristallines Pulver.

\section{Diphenylphenylthiogallan (VII)}

Durchführung und Aufarbeitung wie III. Ansatz: $1.50 \mathrm{~g}(4.98 \mathrm{mmol}) \mathrm{I}$ und 0.550 $\mathrm{g}(5.00 \mathrm{mmol})$ Thiophenol. Ausbeute: $1.55 \mathrm{~g}$ (93.4\%) farbloses, mikrokristallines Pulver.

\section{Diphenylbenzylthiogallan (VIII)}

Durchführung wie III. Nach dem Abziehen des Lösungsmittels wird mit n-Hexan gewaschen und getrocknet. Ansatz: $1.40 \mathrm{~g}(4.65 \mathrm{mmol}) \mathrm{I}$ und $0.578 \mathrm{~g}(4.65 \mathrm{mmol})$ Benzylmercaptan. Ausbeute: $1.60 \mathrm{~g}$ (99.4\%) farbloses, mikrokristallines Pulver. 
Dank

Herrn Prof. Dr. Max Schmidt danken wir für die Ermöglichung dieser Arbeiten, dem Verband der Chemischen Industrie für die finanzielle Unterstützung und dem Rechenzentrum der Universität Würzburg für die Möglichkeit zur Darstellung des ORTEP-Plots.

\section{Literatur}

1 G.E. Coates, J. Chem. Soc., (1951) 2009.

2 G.E. Coates und R.G. Hayter, J. Chem. Soc., (1953) 2519.

3 J.M. Viktorova, N.J. Sheverdina und K.A. Kocheshkov, Dokl. Akad. Nauk, SSSR, Ser. Khim., 198 (1971) 94 (Chem. Abstr., 75 (1971) 49184 a).

4 J. Weidlein, Z. Anorg. Allg. Chem., 386 (1971) 129.

5 G.G. Hoffmann, Z. Naturforsch., im Druck.

6 G.G. Hoffmann, Z. Anorg. Allg. Chem., im Druck und dort zitierte Literatur.

7 A.N. Rodionov, N.I. Ruch'eva, J.M. Viktorova, D.N. Shigorin. N.J. Sheverdina und K.A. Kocheshkov, Izv. Akad. Naul, SSSR, Ser. Khim., (1969) 1047 (Chem. Abstr., 71 (1969) 43908).

8 R.G. Pearson, J. Am. Chem. Soc., 85 (1963) 3533.

9 S.J. Rettig, A. Storr und J. Trotter, Canad. J. Chem., 54 (1976) 1278.

10 (a) M.E. Kenney und A.W. Laubengayer, J. Am. Chem. Soc., 76 (1954) 4839; (b) G.S. Smith und J.L. Hoard, J. Am. Chem. Soc., 81 (1959) 3907.

11 Syntex-XTL-Programme Package, Syntex Analytical Instruments, Cupertino. California, USA, 1976.

12 D.T. Cromer und J.T. Waber, International Tables for X-Ray Crystallography Bd. IV, S. 99, Kynoch Press, Birmingham, (1969).

13 F. Zettler und H. Hess, Chem. Ber., 110 (1977) 3943.

14 G.G. Hoffmann und Ch. Burschka, Veroffentlichung in Vorbereitung.

15 T. Halder, H.-D. Hansen und J. Weidlein, Z. Naturforsch. B, 35 (1980) 773.

16 K. Dymock, G.J. Palenik, J. Slezak, C.L. Raston und A.H. White, J. Chem. Soc., Dalton Trans., (1976) 28.

17 W. Schöniger, Mikrochim. Acta, (1956) 869.

18 Komplexometrische Bestimmungen mit Titriplex, E. Merck, Darmstadt.

19 H. Gilman und R.G. Jones, J. Am. Chem. Soc., 62 (1940) 980. 